\title{
The impact of obesity on inflammatory markers used in the assessment of disease activity in rheumatoid arthritis - a cross-sectional study
}

\author{
Ashish Sharma ${ }^{1}$, Ashok Kumar ${ }^{1}$, Alka Jha ${ }^{2}$, Anunay Agarwal ${ }^{1}$, Anoop Misra ${ }^{2}$ \\ ${ }^{1}$ Department of Rheumatology, Fortis Flt. Lt. Rajan Dhall Hospital, New Delhi, India \\ ${ }^{2}$ Centre for Excellence in Diabetes, Obesity and Cholesterol, Fortis Flt. Lt. Rajan Dhall Hospital, New Delhi, India
}

\begin{abstract}
Introduction: Obesity is known to be associated with elevated levels of inflammatory markers. The aim of the study was to assess the confounding effect of obesity on the levels of erythrocyte sedimentation rate (ESR) and C-reactive protein (CRP) in patients with rheumatoid arthritis (RA) in low disease activity state or remission as indicated by clinical disease activity index (CDAI).

Material and methods: Adult RA patients with CDAl less than 10 were divided into two groups: obese and non-obese, based on body mass index. Relevant exclusions were applied to eliminate causes of raised inflammatory markers other than obesity. The difference of CRP and ESR levels between the obese and non-obese groups was analyzed.

Results: Obese patients with RA $(n=85)$ had higher CRP and ESR than non-obese patients $(n=66)$ ( $p$-values 0.008 and 0.000005 , respectively). In addition, obese females with RA had significantly higher CRP and ESR as compared to non-obese females. However, the difference was not significant in males. Twenty-one obese (24.7\%) and two non-obese RA patients (3\%) had elevated CRP (difference of approximately $22 \%$ [24.7 minus 3]). Forty obese (47\%) and 16 non-obese RA patients (24.2\%) had elevated ESR (difference of approximately 23\% [ 47 minus 24.2]). Thus, obesity was the attributable cause of falsely elevated CRP and ESR in $22 \%$ and $23 \%$ of patients, respectively.

Conclusions: About one-fifth of patients with RA, who are actually in low disease activity, may have elevated inflammatory markers, primarily because of obesity. Therefore, elevated CRP and ESR in obese patients with RA should be interpreted with caution because it may lead to unnecessary overtreatment.
\end{abstract}

Key words: obesity, rheumatoid arthritis, C-reactive protein, erythrocyte sedimentation rate.

\section{Introduction}

Rheumatoid arthritis (RA) is a systemic inflammatory disorder in which raised C-reactive protein (CRP) and erythrocyte sedimentation rate (ESR) suggest active disease. Acute phase reactants are also used in monitoring the patients with RA, to assess the response to treatment. Studies have shown that obesity is positively correlated with raised CRP and ESR in the general population $[1,2]$. Acute phase reactants also rise in response to infection and inflammation. They constitute important components of various composite indices for measuring disease activity of RA. Obesity can falsely raise acute phase reactants in obese patients with RA, independent of disease activity [3]. This can lead to falsely high scores of various disease activity tools, which incorporate acute phase reactants, thereby making the true assessment of disease activity difficult.

Published literature shows evidence of correlation of inflammatory markers and obesity in patients with RA, independent of disease activity. None of the studies described the frequency of this confounding effect. We conducted

Address for correspondence:

Ashish Sharma, Department of Rheumatology, Fortis Flt. Lt. Rajan Dhall Hospital, Aruna Asaf Ali Marg, Pocket 1, Sector B, Vasant Kunj, New Delhi, 110070 India, e-mail: ash.blueney@gmail.com

Submitted: 14.01.2020; Accepted: 24.02.2020 
this study to assess how often obesity causes a confounding effect on the raised inflammatory markers in patients with RA in low disease activity state or remission.

The primary objective was to study the frequency of the confounding effect of obesity on the levels of CRP and ESR in patients with rheumatoid arthritis in low disease activity state or remission, using the clinical disease activity index (CDAI). The effect of gender on CRP and ESR levels in RA patients was also studied as a secondary objective.

\section{Material and methods}

Adult RA patients (2010 American College of Rheumatology criteria) aged more than 18 years and with CDAI $<10$ (CDAl is the sum of the number of tender joints, number of swollen joints, physician global assessment [0 to 10], and patient global assessment [0 to 10]) were recruited into the study from January 2018 to December 2018, after obtaining informed consent $[4,5]$. A sample size of 144 subjects (type I error 5\% and type II error 20\%, Z-value 1.96, confidence level 95\%) was obtained with the assumption that elevation of CRP and ESR attributable to obesity would occur in at least $10 \%$ of patients with RA in low disease activity state/remission (as defined by CDAI). Low disease activity was defined by CDAI of 2.8 to 9 and remission at CDAl below 2.8.

Patients were divided into two groups: group A comprised obese RA patients defined by a body mass index (BMI) of > 25 [6]. Group B comprised non-obese RA patients (BMI < 25). A third control group comprising obese individuals without RA was also included in the study. CRP levels and ESR were obtained on all study subjects. CRP (in milligrams per liter; $\mathrm{mg} / \mathrm{l}$ ) was measured by nephelometry and ESR (in millimeters per hour; $\mathrm{mm} / \mathrm{h}$ ) by Westergren's method.

Exclusions comprised patients with any evidence of infection currently or in the past month, major surgery in the past three months, another inflammatory disorder (e.g. interstitial lung disease, chronic obstructive pulmonary disease, scleritis/episcleritis, rheumatoid vasculitis, overlap with another connective tissue disease), pregnancy, anemia (hemoglobin < $10 \mathrm{~g} / \mathrm{dl}$ ), current smoking, polycythemia, sickle cell anemia, liver disease, or malignancy. Patients treated with tocilizumab were excluded from this analysis; patients treated with all other biologic disease-modifying antirheumatic drugs (DMARDs) were included in the study if they were having remission or low disease activity state. Abatacept is not available in India anymore, so none of our patients were treated with this drug.

It was a cross-sectional study. However, study groups were not matched for age and gender. Statistical significance of the difference of CRP and ESR levels between groups $A$ and $B$, between group $A$ and controls, and between group $B$ and controls was analyzed by Mann-Whitney $U$ test. Statistical significance was set at $p \leq 0.05$

Informed consent was obtained from patients included in the study, and ethical clearance was obtained from our hospital's institutional review board. Date of approval: 27 ${ }^{\text {th }}$ August 2019, No. ECR/TH/2019/04.

\section{Results}

Eighty-five obese RA patients (12 males [M], 73 females [F]), 66 non-obese RA patients (17 M, 49 F), and 52 obese controls (16 M, 36 F) were recruited into the study (Table I).

Table I. Baseline characteristics

\begin{tabular}{|c|c|c|c|}
\hline Parameters & $\begin{array}{l}\text { Obese RA } \\
(n=85)\end{array}$ & $\begin{array}{c}\text { Non-obese RA } \\
(n=66)\end{array}$ & $\begin{array}{l}\text { Obese controls } \\
\quad(n=52)\end{array}$ \\
\hline Mean age (years) & 55.165 & 51.061 & 48.1 \\
\hline Number of males (\%) & $12(14.1)$ & $17(25.8)$ & $16(30.8)$ \\
\hline Number of females (\%) & $73(85.9)$ & $49(74.2)$ & $36(69.2)$ \\
\hline Mean disease duration (months) & 110.75 & 74.9 & \\
\hline Mean BMI $\left(\mathrm{kg} / \mathrm{m}^{2}\right)$ & 29.5 & 21.6 & 33.5 \\
\hline Median CDAI (IQR) & $0(5)$ & $0(4)$ & \\
\hline Median CRP (IQR) & $5(5.7)$ & $3(3)$ & $3.45(7.2)$ \\
\hline Median ESR (IQR) & $30(21)$ & $18(19.5)$ & $20.5(20)$ \\
\hline Mean CDAI (SD) & $2.53(3.16)$ & $2.07(2.8)$ & \\
\hline Mean CRP (SD) & 9.9 (13.01) & $4.7(7.96)$ & $5.4(4.26)$ \\
\hline Mean ESR (SD) & $30.7(17.13)$ & $20.4(13.01)$ & $25.7(17.43)$ \\
\hline
\end{tabular}

$R A$ - rheumatoid arthritis, $B M I$ - body mass index, CDAI - clinical disease activity index, IQR - interquartile range, CRP-C-reactive protein, $E S R$ - erythrocyte sedimentation rate, SD - standard deviation. 
Among all female patients with RA ( $n=122)$, 91 were post-menopausal (74.5\%). Mean BMI was 29.5, 21.6, and $33.5 \mathrm{~kg} / \mathrm{m}^{2}$ in the three groups, respectively. Obese and non-obese RA patients had median CDAl of 0 (IQR 5 and 4, respectively). Fifty-two obese RA patients (61.1\%) and 43 non-obese RA patients (65.1\%) were in remission (Fig. 1). Median CRP levels in the 3 groups were 5 (IQR 5.7), 3 (IQR 3) and 3.45 (IQR 7.2) respectively and median ESR was 30 (IQR 21), 18 (IQR 19.5) and 20.5 (IQR 20) respectively. Obese patients with RA had mean CRP of 7.82 (95\% confidence interval $7.82 \pm 1.82$ ) and mean ESR of 32.8 (95\% confidence interval $32.8 \pm 3.73$ ). Non-obese patients with RA had mean CRP of 3.88 (95\% confidence interval $3.88 \pm 0.74)$ and mean ESR of 21.19 (95\% confidence interval $21.19 \pm 3.45)$. Among patients with RA, obese patients had significantly higher CRP and ESR than non-obese patients ( $p$-values 0.008 [effect size 0.2984 ] and 0.000005 [effect size 0.3466], respectively) (Fig. 2). The difference in CRP level between obese RA patients and obese controls was not significant ( $p$-value 0.2 [effect size 0.283]).

The difference in CRP and ESR between obese and non-obese RA patients was not significant for males ( $p$-value 0.964 [effect size 0.0815] and 0.051 [effect size $0.2841]$, respectively), unlike for females ( $p$-value 0.008 [effect size 0.324] and 0.0001 [effect size 0.34], respectively) (Fig. 2). CRP levels did not differ significantly between males and females in the three groups. The same was true for ESR in obese RA and non-obese RA patients. In contrast, the difference in ESR between females and males in the obese control group just reached statistical significance (median 21.5 and 16, respectively; $p$-value 0.048 [effect size 0.2384]).

Twenty-one obese RA patients (24.7\%) and two non-obese RA patients (3\%) had CRP levels more than $10 \mathrm{mg} / \mathrm{l}$ (difference of approximately 22\% between the two groups [24.7 minus 3]). Similarly, 40 obese RA patients (47\%) and 16 non-obese RA patients (24.2\%) had ESR more the $30 \mathrm{~mm} /$ hour (difference of approximately $23 \%$ between the two groups [ 47 minus 24.2]). Thus, obesity contributed to falsely elevated CRP and ESR in $22 \%$ and $23 \%$ of patients with RA, respectively (Fig. 3 ).

\section{Discussion}

The present study showed that obese patients with RA had a significantly higher CRP and ESR than non-obese RA patients. When CRP of obese RA patients with low disease activity/remission was compared with that of obese non-RA controls, there was no significant difference. Here the CRP elevation was accounted by obesity mainly. Interestingly, obesity was not associated with significantly higher CRP and ESR in males with RA in our study.

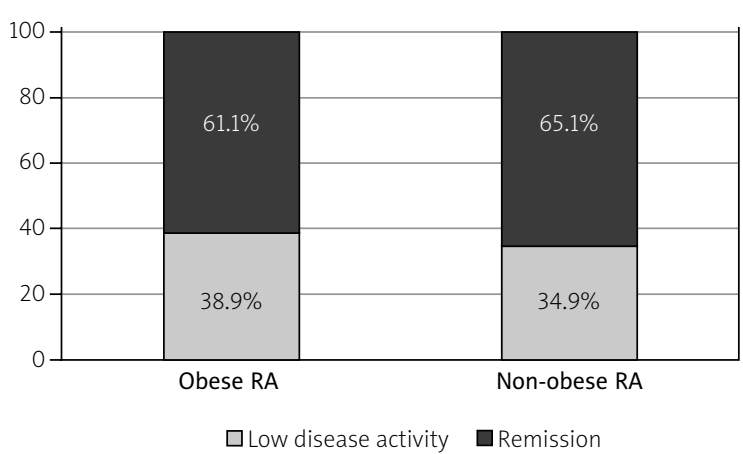

Fig. 1. Disease activity state in patients with rheumatoid arthritis (RA).

George et al. [3] reported similar observations, in which they showed that CRP and ESR positively correlated with obesity in females with RA but not in males. It was a large study in which the association of CRP and ESR with obesity was studied in two RA cohorts and a control cohort without RA. Dual-energy X-ray absorptiometry (DEXA) was used to assess the truncal fat mass. Positive correlation between CRP and BMI was found in females with and without RA. Similar correlation was found between ESR and BMI in females. The association between CRP and BMI vanished when fat mass was adjusted, thus confirming that CRP correlated mainly with fat mass. Adipose tissue is a site for synthesis of many mediators of inflammation, including interleukin 6 (IL-6) [7]. This leads to an increase in acute phase reactants in obese individuals. Weight loss due to inflammation because of active disease was hypothesized by George et al. to be the cause of poor association of CRP and ESR with $\mathrm{BMI}$ in men with $\mathrm{RA}$ [3].

Testosterone seems to have an inhibitory effect on CRP because lower CRP levels are found in males with higher testosterone levels [8].

Fat around internal organs (visceral fat) has a greater effect on CRP synthesis by liver, as compared to truncal fat. In all the previous studies, truncal fat was estimated by DEXA. Assessment of visceral fat by computed tomography (CT) of the abdomen may remove the discrepancy between obesity and acute phase reactants observed in males with RA.

Among the anthropometric parameters, waist circumference has been shown to correlate most closely with CRP in the healthy population [9]. This is also true in patients with RA, independent of disease activity [10]. Higher levels of CRP and IL- 6 were found in women with RA with a higher BMI and truncal fat measured by DEXA [11]. In the same study, treatment with biological drugs did not alter the positive correlation between CRP and 
A

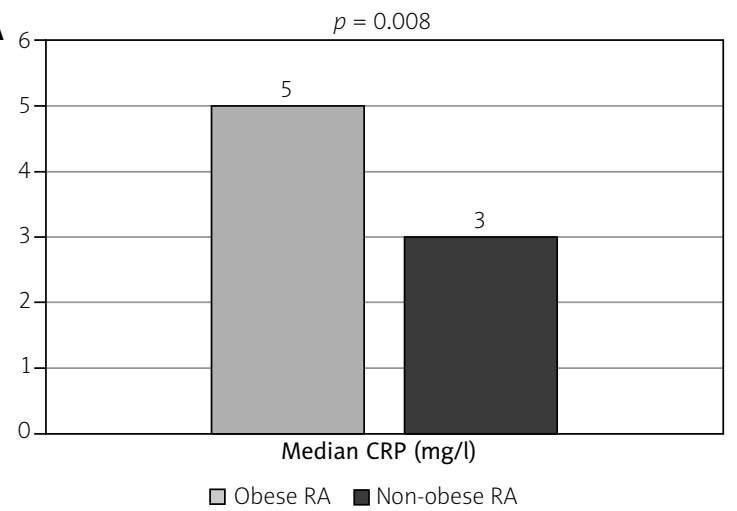

C

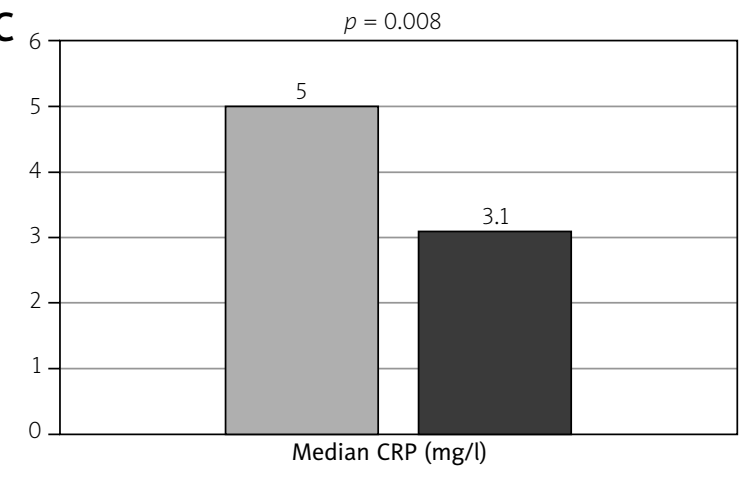

$\square$ Obese RA females

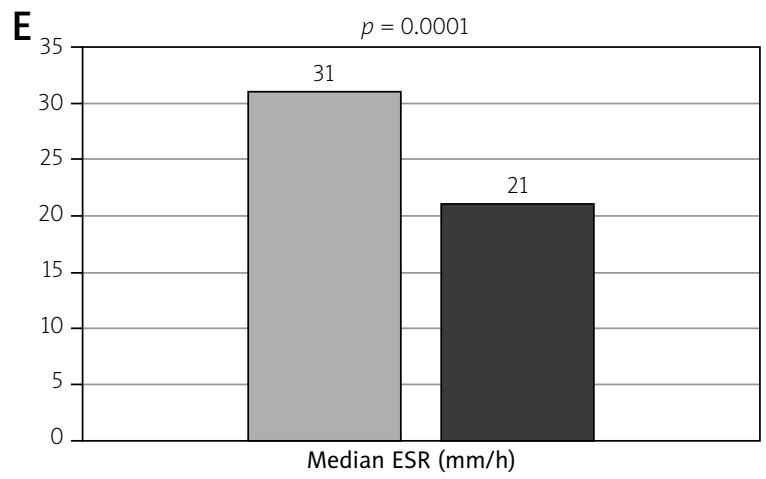

$\square$ Obese RA females

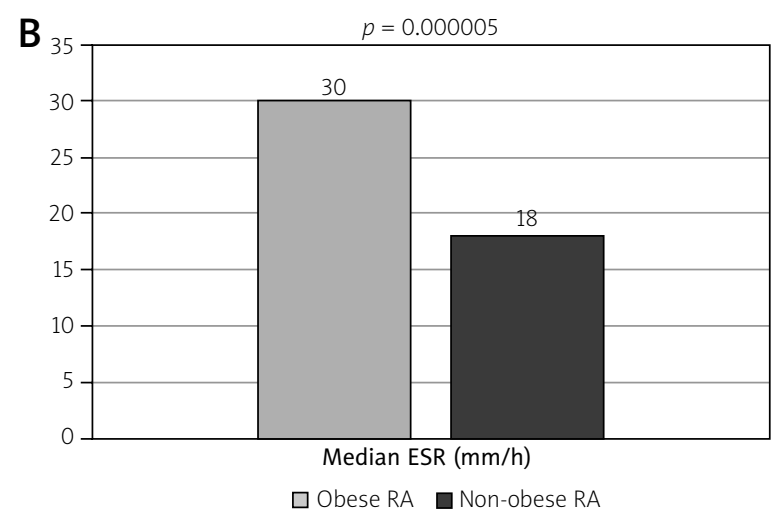

$\mathbf{D}_{3.5}$

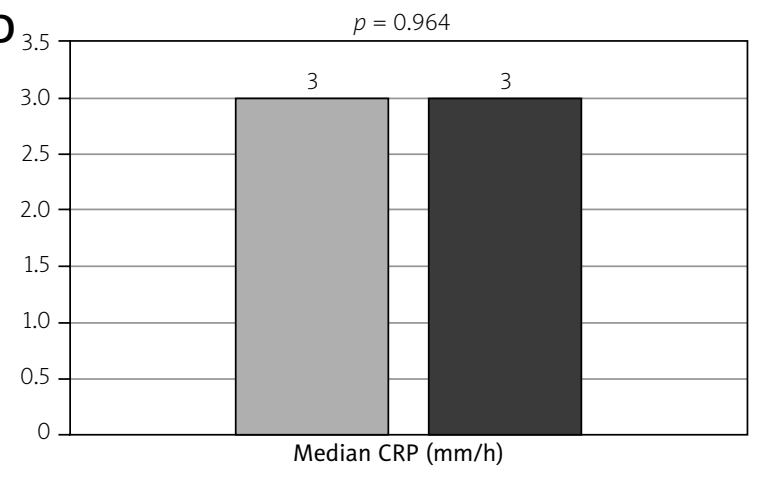

$\square$ Obese RA males $\square$ Non-obese RA males

$\mathbf{F}$

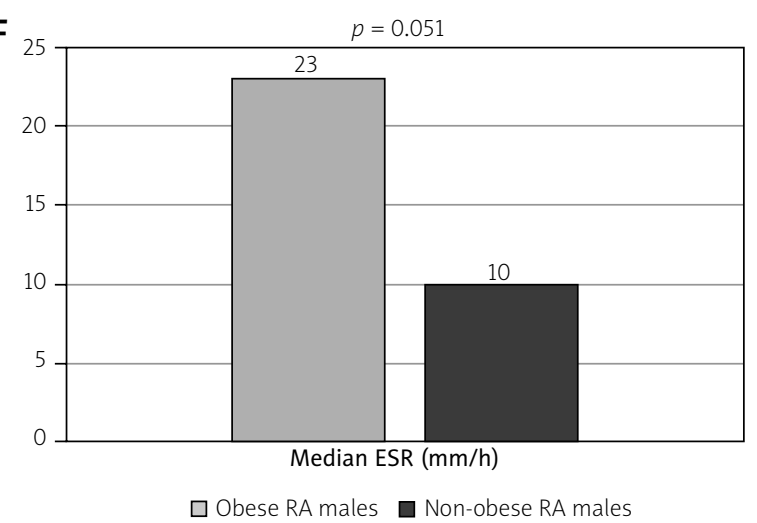

Fig. 2. A - C-reactive protein (CRP) levels in patients with rheumatoid arthritis (RA), B - erythrocyte sedimentation rate (ESR) values in patients with RA, $C-C R P$ levels in female patients with RA, D - CRP levels in male patients with RA, E - ESR values in female patients with RA, F-ESR values in male patients with RA.

truncal fat. In addition, a similar correlation between obesity and IL-6 levels was observed, as with CRP.

None of the studies mentioned above included patients in low disease activity or remission. However, adjustment for disease activity was made in these studies. We took patients in low disease activity or remission, to effectively rule out active disease as a cause for the raised acute phase reactants. However, subclinical inflammation might be present in some patients, which could have contributed to raised CRP or ESR. Ultrasound or magnetic resonance imaging can detect subclinical inflammation [12, 13].
The confounding effect of obesity on inflammatory markers is not well studied in patients with other rheumatic diseases. However, Oeser et al. [14] showed that obese patients with SLE had significantly higher levels of CRP and ESR.

Raised markers of inflammation should be interpreted with caution in obese patients with RA, because it can have implications on the treatment. In addition, obese patients tend to report higher scores of subjective disease activity [15]. These factors may result in unnecessary overtreatment of many obese patients by 
$\mathrm{A}_{30}$

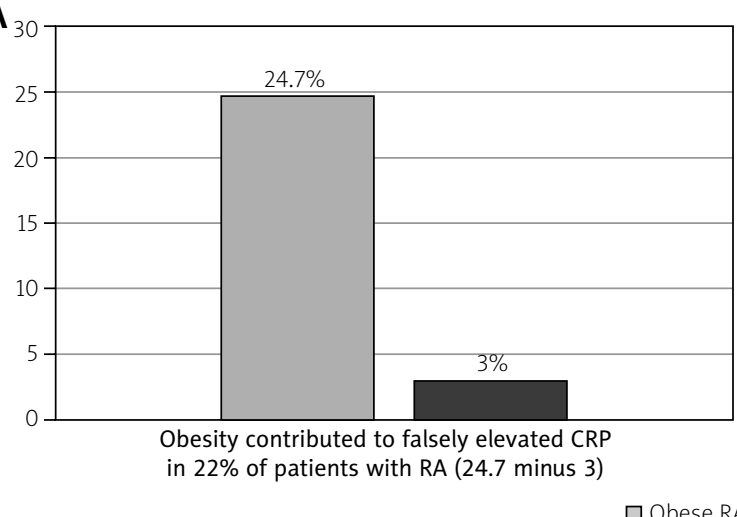

B

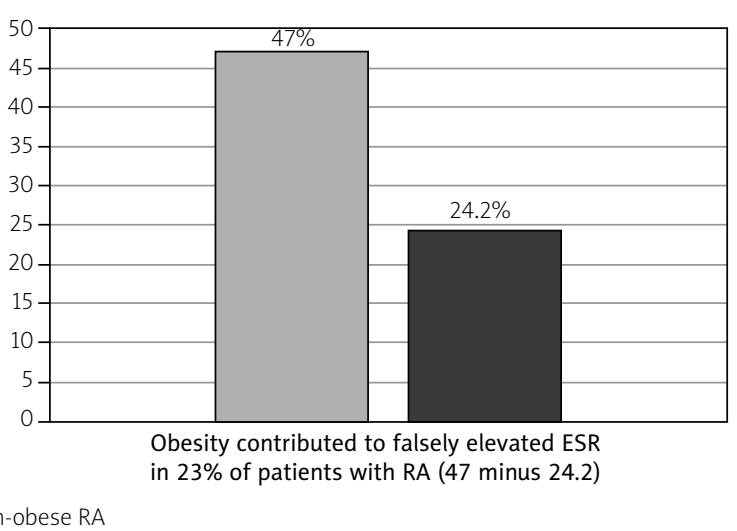

Fig. 3. Percentage of rheumatoid arthritis (RA) patients having C-reactive protein (CRP) more than $10 \mathrm{mg} / \mathrm{l}$ (A) and erythrocyte sedimentation rate (ESR) more than $30 \mathrm{~mm} / \mathrm{h}$ (B).

a "treat-to-target" approach due to falsely high disease activity scores on the composite indices dependent on acute phase reactants and patients' global assessment. In the current era, where there are multiple options for the treatment of RA, judicious use of drugs is necessary to avoid overtreatment.

We recruited patients with RA with low disease activity or remission, thereby eliminating disease activity as the cause for elevated CRP or ESR. Moreover, using strict exclusion criteria, we eliminated other potential contributors to elevated inflammatory markers and excluded patients treated with tocilizumab because it has a direct effect of reduction in the levels of CRP by reducing its synthesis in liver. One limitation of our study was the lack of objective assessment of fat mass by DEXA or visceral fat mass (by CT abdomen). It is noteworthy that imaging techniques for assessment of body fat do not have standardized definitions for obesity.

\section{Conclusions}

Obesity may falsely elevate CRP and ESR in as many as $22 \%$ and $23 \%$, respectively, of RA patients with low disease activity. Clinicians should be cautious while treating RA patients with a "treat-to-target" approach, in which composite disease activity indices utilizing acute phase reactants as one of their components are used. In the current era, when there are multiple options for the treatment of RA (including biological DMARDs), judicious use of drugs is necessary to avoid overtreatment.

\section{Acknowledgements}

The authors would like to thank Dr. Anoushka Kapila for her valuable input in this study.

The authors declare no conflict of interest.

\section{References}

1. Vallianou NG, Evangelopoulos AA, Panagiotakos DB, et al. Associations of acute-phase reactants with metabolic syndrome in middle-aged overweight or obese people. Med Sci Monit 2010; 16: CR56-60.

2. Aronson D, Bartha P, Zinder O, et al. Obesity is the major determinant of elevated $C$-reactive protein in subjects with the metabolic syndrome. Int J Obes Relat Metab Disord 2004; 28: 674-679, DOI: 10.1038/sj.ijo.0802609.

3. George MD, Giles JT, Katz PP, et al. Impact of Obesity and Adiposity on Inflammatory Markers in Patients With Rheumatoid Arthritis. Arthritis Care Res (Hoboken) 2017; 69: 1789-1798, DOI: 10.1002/acr.23229.

4. Aletaha D, Neogi T, Silman AJ, et al. 2010 Rheumatoid arthritis classification criteria: an American College of Rheumatology/ European League Against Rheumatism collaborative initiative. Arthritis Rheum 2010; 62: 2569-2581, DOI: 10.1002/art.27584.

5. Aletaha D, Nell VPK, Stamm T, et al. Acute phase reactants add little to composite disease activity indices for rheumatoid arthritis: validation of a clinical activity score. Arthritis Res Ther 2005; 7: R796-R806, DOI: 10.1186/ar1740.

6. Misra A, Chowbey P, Makkar BM, et al. Consensus statement for diagnosis of obesity, abdominal Obesity and the metabolic syndrome for Asian Indians and recommendations for physical activity, medical and surgical management. J Assoc Physicians India 2009; 57: 163-170.

7. Pou KM, Massaro JM, Hoffmann, et al. Visceral and subcutaneous adipose tissue volumes are cross-sectionally related to markers of inflammation and oxidative stress: the Framingham Heart Study. Circulation 2007; 116: 1234-1241, DOI: 10.1161/CIRCULATIONAHA.107.710509.

8. Kapoor D, Clarke S, Stanworth R, et al. The effect of testosterone replacement therapy on adipocytokines and C-reactive protein in hypogonadal men with type 2 diabetes. Eur J Endocrinol 2007; 156: 595-602, DOI: 10.1530/EJE-06-0737.

9. Faam B, Zarkesh M, Daneshpour SM, et al. The association between inflammatory markers and obesity-related factors in Tehranian adults: Tehran lipid and glucose study. Iran J Basic Med Sci 2014; 17: 577-582. 
10. Dessein PH, Norton GR, Woodiwiss AJ, et al. Independent role of conventional cardiovascular risk factors as predictors of C-reactive protein concentrations in rheumatoid arthritis. J Rheumatol 2007; 34: 681-688.

11.Giles JT, Bartlett SJ, Andersen R, et al. Association of body fat with C-reactive protein in rheumatoid arthritis. Arthritis Rheum 2008; 58: 2632-2641, DOI: 10.1002/art.23766.

12. Hammer HB, Kvien TK, Terslev L. Ultrasound of the hand is sufficient to detect subclinical inflammation in rheumatoid arthritis remission: a post hoc longitudinal study. Arthritis Res Ther 2017; 19: 221, DOI: 10.1186/s13075-017-1428-4.

13.Zhang $\mathrm{H}, \mathrm{Xu} \mathrm{H}$, Chen S, Mao X. The application value of MRI in the diagnosis of subclinical inflammation in patients with rheumatoid arthritis in remission. J Orthop Surg Res 2018; 13 : 164, DOI: 10.1186/s13018-018-0866-2.
14. Oeser A, Chung CP, Asanuma Y, et al. Obesity is an independent contributor to functional capacity and inflammation in systemic lupus erythematosus. Arthritis Rheum 2005; 52: 3651-3659, DOI: 10.1002/art.21400.

15. Liu Y, Hazlewood GS, Kaplan GG, et al. The Impact of Obesity on Remission and Disease Activity in Rheumatoid Arthritis: A Systematic Review and Meta-Analysis. Arthritis Care Res (Hoboken) 2017; 69: 157-165, DOI: 10.1002/acr.22932. 